\title{
Off-resonance field enhancement by spherical nanoshells
}

\author{
Andrey E. Miroshnichenko* \\ Nonlinear Physics Centre, Research School of Physics and Engineering, Australian National University, \\ Canberra Australian Capitol Territory 0200, Australia
}

(Received 20 December 2009; revised manuscript received 23 February 2010; published 11 May 2010)

\begin{abstract}
We study light scattering by spherical nanoshells consisting of metal-dielectric composites. We consider several geometries of nanoshell particles with dielectric and metallic cores. We demonstrate that in all studied cases the local field enhancement takes place in out-of-resonance regions ("dark states"), which nevertheless can be understood in terms of the Fano resonance. At optimal conditions the light is mostly localized inside the dielectric material and the enhancement factor up to four orders of magnitude is achieved for subwavelength coating. Using nonlinear dielectric materials will lead to a variety of nonlinear phenomena applicable for photonics applications.
\end{abstract}

DOI: 10.1103/PhysRevA.81.053818

PACS number(s): 42.25.Bs, 42.25.Fx, 78.67.Bf

\section{INTRODUCTION}

Electromagnetic properties of metallic nanoparticles have attracted much attention during the last decade due to their ability to squeeze light at the subwavelength level. Coherent excitation of conduction electrons on a metallic surface allows for the enhancement of the optical field close to the surface of the particle. Such local field enhancement was used for sensing, single molecule detection, surface-enhanced Raman scattering, medicine, biology, and other applications [1]. The understanding of how metallic geometries control the properties of surface plasmons will lead to a precise design of metallic nanoparticles for optimized optical field enhancement. One of the successful examples is metallic nanoshell structures with a dielectric core. For such structures, plasmon hybridization theory was developed $[2,3]$. In the framework of this theory, plasmons of adjacent surfaces of complex metallic geometries may interact and therefore hybridize in analogy with the wave functions of a quantum system. The unique property of metallic nanoshell structures is the sensitivity of surface plasmons to the thickness of the metallic shell. It allows us to design materials to match the required wavelength for a particular application in visible or near-infrared regions.

Despite such broad tunability, the existence of the inherent optical losses of metals prevents them from having a successful application in photonics so far. Recently, it was experimentally demonstrated that the conjugate geometry with a metallic core and dye-doped coated dielectric may allow us to overcome the loss-of-surface plasmon resonances [4]. It may lead to a first realization of a spaser (surface plasmon amplification by stimulated emission of radiation) nanoplasmonic counterpart of the laser, first predicted in Ref. [5]. The spaser-based nanolaser may become a key component for future nanophotonic technologies.

In this paper we investigate several geometries of coated nanoparticles with dielectric and metallic cores embedded into a dielectric medium. For our analysis we consider silver-silicon composites in air. The main attention is paid to the local field enhancement inside and near the nanoparticle. One of the common approaches to analyzing field enhancement is by means of scattering resonances of a coated sphere. The

\footnotetext{
*aem124@physics.anu.edu.au
}

$Q$ factor of a resonance is associated with the amount of the field which can be stored inside the nanoparticle. However, our analysis reveals that maximal field enhancement does not necessarily coincide with the enhanced scattering. On the contrary, we demonstrate that, in some cases, the maximal enhancement may correspond to resonant suppression of the scattering. These so-called "dark states" correspond to the strong local excitation of the electromagnetic field, which does not couple to the far field. Such behavior can be interpreted in terms of the Fano resonances. The use of a high-refractiveindex dielectric allows us to localize the electric field inside the nonabsorbing layer. We found that the most promising structure is the metallic core coated with a thin layer of a low-refractive-index dielectric embedded into a high-index dielectric. The enhanced light power inside the nonlinear dielectric layer may potentially lead to a nonlinear response of the whole structure. This could be another approach to overcome the losses of metals and the use of metallic nanoparticles in photonic applications.

\section{LOCALIZED SURFACE PLASMON RESONANCES}

Light scattering by a spherical particle is one of the most elaborate problems in electromagnetic theory. The analytical solution was found independently more than a hundred years ago by Lorenz [6] and Mie [7]. Although this solution has been known for a very long time and was analyzed from different perspectives, it still contains some hidden results [8]. According to the Mie theory of light scattering by dielectric spheres [9], the positions of resonances can be characterized by the size parameter $x=2 \pi a / \lambda$, where $a$ is the radius of the sphere and $\lambda$ is the input wavelength. Thus for larger spheres there is a linear redshift of Mie resonances with the radius of the sphere.

In metallic nanoparticles, the incident light can couple to free electrons of the conduction band on the surface of the metal. Such excitations are known as localized surface plasmon resonances (LSPR), and are usually associated with the enhancement of the electric field near the surface [9]. According to the Mie solution in the small particle limit $a \ll \lambda$, the condition for LSPR can be written as follows

$$
\operatorname{Re}\left[\epsilon_{m}\left(\omega_{\mathrm{LSPR}}\right)\right]=-\frac{l+1}{l} \epsilon_{o},
$$


where $\epsilon_{m}$ and $\epsilon_{o}$ are permittivities of the metal and surrounding dielectrics, and $l=1,2,3, \ldots$ is the mode number corresponding to the dipole, quadrupole, octupole, and so on resonances. One of the distinguishing features of the surface modes is that the internal electric field does not have a radial node inside the metal [9]. Due to the dispersion of a metal, condition (1) can be satisfied for a number of frequencies for the same metal. Although the absorption of metals can be relatively small $\left|\operatorname{Im}\left[\epsilon_{m}(\omega)\right]\right|<1$, the absorption efficiency of the metallic nanoparticle in the vicinity of LSPR's can be effectively very large $Q_{\mathrm{abs}} \propto 1 / \operatorname{Im}\left[\epsilon_{m}\left(\omega_{\mathrm{LSPR}}\right)\right]$. As a result, the scattering properties are greatly modified at LSPR's. Recently, it was predicted that weakly absorbing particles may exhibit an inverse hierarchy of optical resonances [10]. It results in narrower resonances for higher-order modes $l=1,2,3, \ldots$. with a possibility to store more energy inside due to a larger $Q$ factor [11].

\section{LIGHT SCATTERING BY NANOSHELL PARTICLES}

The main focus of this paper is the electric field enhancement by coated nanoparticles in dielectric-metal composites [see Figs. 1(a)-1(c)]. The conventional Lorenz-Mie theory has been extended by Aden and Kerker [12] for the scattering by a coated sphere with arbitrary parameters. The complete solution consists of an infinite series of Bessel's function, which can be truncated depending on the particle size [9]. It still requires the use of a numerical approach to be fully analyzed. Up to now, many special algorithms for fast and accurate calculations of Bessel's functions and their derivatives were developed [13].

We perform the comparative analysis of three different geometries of a coated small particle in dielectric-metal composites. The first geometry is the metallic spherical shell with a dielectric core in air [see Fig. 1(a)]. The second and third geometries are a metallic nanoparticle coated with high-refractive-index [see Fig. 1(b)] and low-refractive-index [see Fig. 1(c)] dielectric layers with respect to the surrounding medium. For our study, without loss of generality, we consider $\mathrm{Si}$-Ag-air composites. For silicon we take the refractive index to be $n=3.4$, and for silver we use experimental data [14] with a small-size Drude model correction $[15,16]$.

The condition for excitation of the first-order surface mode $l=1$ for coated spherical nanoparticles can still be obtained analytically in the small particle limit $b \ll \lambda[9]$

$\left(\epsilon_{b}+2 \epsilon_{o}\right)\left(\epsilon_{a}+2 \epsilon_{b}\right)+\left(\frac{a}{b}\right)^{3}\left(2 \epsilon_{b}-2 \epsilon_{o}\right)\left(\epsilon_{a}-\epsilon_{b}\right)=0$,

where $\epsilon_{a}$ and $\epsilon_{b}$ are permittivities of the core and mantle of radii $a$ and $b$, respectively. According to this equation for the first geometry of a silver nanoshell with a silicon core $\epsilon_{a}=\epsilon_{\mathrm{Si}}$ and $\epsilon_{b}=\epsilon_{\mathrm{Ag}}(\omega)$ there are two LSPR's-surfacelike with an external medium and voidlike with an internal dielectric. In general, these two modes are excited at different frequencies, but there was a study demonstrating that under certain conditions they may overlap [17,18]. For the second and third geometries of the dielectric-coated silver nanoparticle with $\epsilon_{a}=\epsilon_{\mathrm{Ag}}(\omega)$ there is a single first-order surface mode. By varying the thickness of the coating layer $d=b-a$, the LSPR frequency of the coated silver lies in the region $-2 \epsilon_{b, o}<\epsilon_{\mathrm{Ag}}\left(\omega_{\mathrm{LSPR}}\right)<-2 \epsilon_{o, b}$ depending on the ratio $\epsilon_{b} / \epsilon_{o}$.
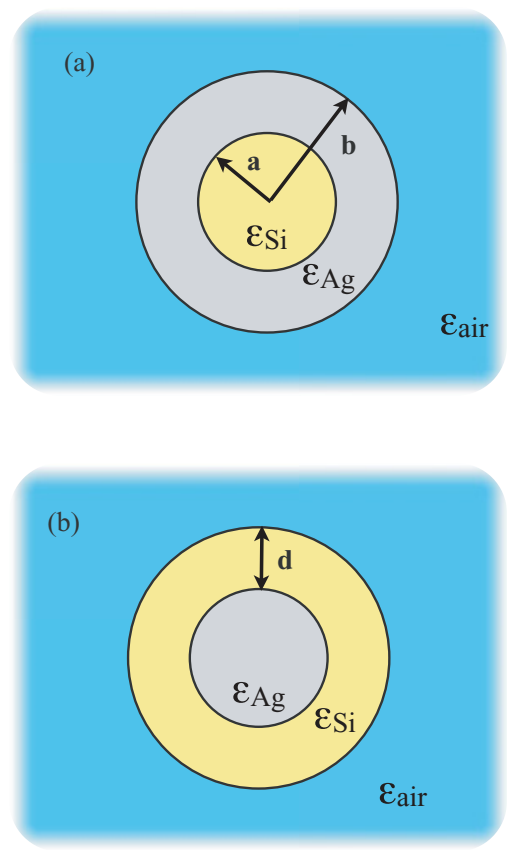

(c)

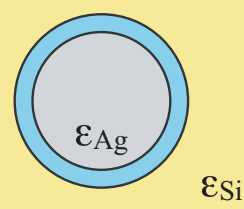

FIG. 1. (Color online) Schematic view of considered geometries: (a) silver nanoshell with silicon core embedded in air; (b) siliconcoated silver embedded in air; and (c) metallic nanoparticle coated by low-refractive-index dielectric (air or polymer) embedded in silicon.

This result indicates that the coating layer allows us to shift effectively the LSPR frequency in a very broad range.

In realistic systems, surface modes can be detected via scattering resonances. According to the Lorenz-Mie theory, scattering by spherical particles is described by the extinction efficiency [9]

$$
Q_{\text {ext }}=Q_{\text {abs }}+Q_{\text {sca }},
$$

where $Q_{\text {sca }}$ and $Q_{\text {abs }}$ are the scattering and absorption efficiencies. As was mentioned earlier, at LSPR frequncies absorption by metallic nanoparticles can be greatly enhanced. For our studies we will use the total extinction $Q_{\text {ext }}$ and absorption $Q_{\text {abs }}$ efficiencies since they contain all the needed information and the scattering efficiency can be derived from (3).

\section{A. Metallic nanoshell}

We start our consideration from the case of light scattering by a silver nanoshell with a silicon core in air. The results for this geometry are summarized in Fig. 2. The radius 

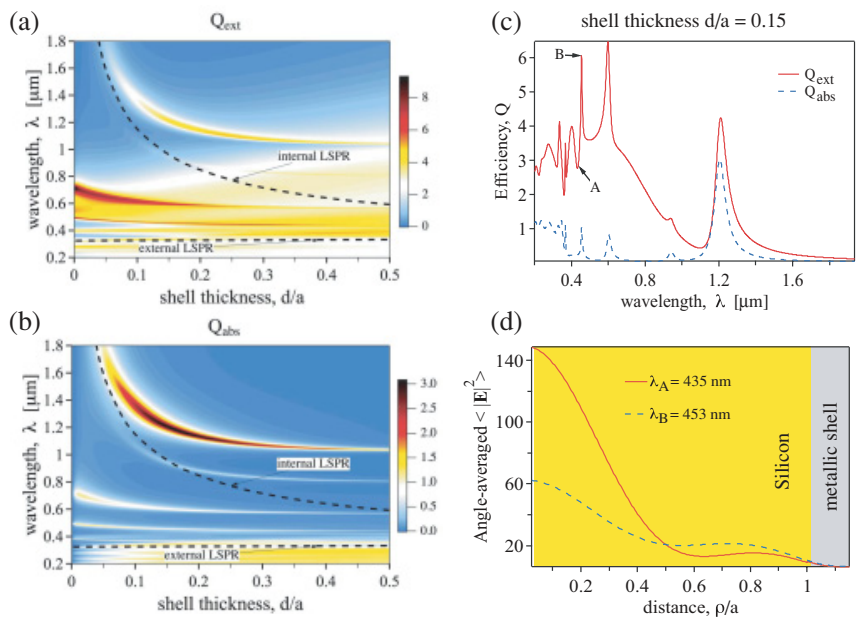

FIG. 2. (Color online) Scattering by the silver nanoshell with a silicon core of radius $a=100 \mathrm{~nm}$ in air. (a) Extinction $Q_{\text {ext }}$ and (b) absorption $Q_{\text {abs }}$ efficiencies versus incident wavelength $\lambda$ and normalized shell thickness $d / a$. Dashed lines correspond to first-order internal and external LSPR modes for small particle limit (2); (c) scattering for $d=15 \mathrm{~nm}$ shell thickness; (d) angle-averaged electric field profile inside the nanoshell for two particular wavelengths (i) out of resonance $\lambda_{A}$, and (ii) at the resonance $\lambda_{B}$, marked in plot (c).

of the silicon core is fixed to $a=100 \mathrm{~nm}$, and the silver shell thickness varies up to $d=50 \mathrm{~nm}$. Both extinction and absorption efficiencies saturate for larger shell thickness because the optical skin depth of silver is less than $d_{S}<40 \mathrm{~nm}$ [see Figs. 2(a) and 2(b)]. The dashed lines in these plots indicate the location of first-order voidlike and surfacelike LSPR modes according to Eq. (2). They are in good agreement for small shell thickness, where the perturbation analysis is still valid. Although, they deviate from the exact solution, the general trend of the first-order mode dependence can be qualitatively grasped. In addition to the first order there are higher-order modes. The wavelength dependence of internal and external LSPR modes is different. Voidlike and surfacelike modes exist below and above the plasma frequency of silver $\lambda_{p} \approx 320 \mathrm{~nm}$, respectively. For that particular geometry, we do not observe overlapping of different modes, which will require passing one of the modes through the plasma frequency [19]. Note here that maximal extinction and absorption resonances correspond to the excitation of voidlike internal modes [20,21] but take place for different modes numbers. Absorption is maximal for the dipole internal LSPR mode $l=1$, while extinction is maximal for the quadrupole LSPR mode $l=2$ (as is the scattering efficiency) for the shell thickness less than the penetration depth $d<d_{S}$. This result indicates that the scattering by metallic nanoshell becomes stronger by accumulating light inside the dielectric core.

There is a common belief that at stronger scattering resonances the light field is enhanced within or in the vicinity of the nanoparticle. However, according to this study it is not always true. To demonstrate this we consider light scattering at a fixed shell thickness $d=15 \mathrm{~nm}$ [see Fig. 2(c)]. By calculating the angle-averaged electric field intensity $\left\langle|\mathbf{E}|^{2}\right\rangle$ inside the nanoshell [22] for this shell thickness we found that the maximal field enhancement corresponds to the resonant suppression of the extinction [see Fig. 2(d)]. This kind of excitation can be called a "dark state" where the excited local field effectively does not emit any background radiation. Meanwhile at the nearest octupole resonance the field enhancement is three times less. Such behavior can be easily understood in terms of the Fano resonance. Indeed, recently it was demonstrated that the light scattering by weakly absorbing particles exhibits asymmetric scattering line shapes [23], which is applicable to metal nanoshell structures as well. The asymmetry comes from the constructive and destructive interference of the incident and reemitted light of an LSPR mode. Such asymmetric profiles were described for the first time by Fano in his seminal paper [24], where he derived formulas for such kinds of resonances, which were named after him

$$
F(\epsilon)=\sigma_{0} \frac{(\epsilon+q)^{2}}{1+\epsilon^{2}}+\sigma_{\mathrm{bg}},
$$

with $\epsilon=2\left(E-E_{R}\right) / \Gamma$ where $E_{R}$ and $\Gamma$ are the position and width of the resonance, $\sigma_{0}$ and $\sigma_{\mathrm{bg}}$ are the normalized and background scattering, and $q$ is the asymmetry parameter. According to this formula, maximal and minimal scattering (constructive and destructive interference) take place at $E_{\max }=E_{R}+\Gamma /(2 q)$ and $E_{\min }=E_{R}-\Gamma q / 2$, respectively, while the actual resonance frequency is somewhere in between, depending on the asymmetry parameter $q$ [25]. It allows us to conclude that the maximal scattering might not necessarily correspond to the excitation of a resonant mode, which also explains the lack of maximal field enhancement. To prove that we are dealing with the Fano resonance in this case, we will fit the scattering with the Fano formula (4). Since the extinction efficiency $Q_{\text {ext }}$ is averaged among all possible directions and polarizations, the resonance properties are averaged as well. Therefore, we will look at the scattering at the particular direction, namely, at the backscattering cross section $Q_{b}$, which is polarization independent. The resulting backscattering in the vicinity of the resonance and its Fano fit is presented in Fig. 3. In the figure one can clearly see that the Fano formula accurately describes positions of the local minima and maxima, indicating that this is indeed the Fano resonance. The discrepancy between the actual and the fitting curves comes from the fact that there are other resonances, which change asymptotic behavior. The main conclusion

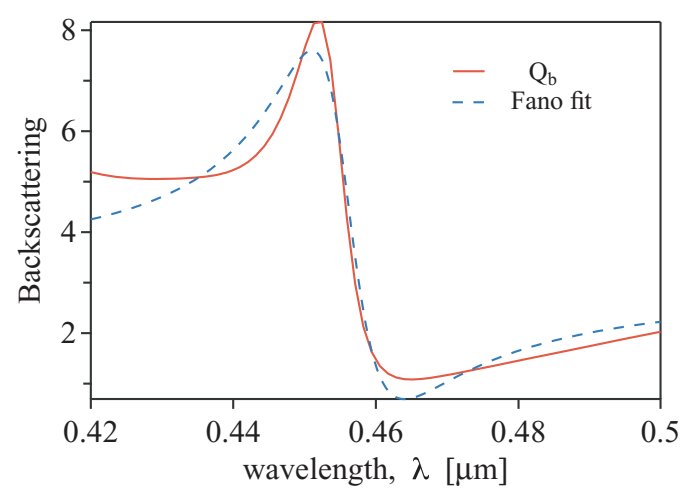

FIG. 3. (Color online) Calculated backscattering cross section $Q_{b}$ and its fitting with the Fano formula (4) in the vicinity of wavelengths $\lambda_{A, B}$ in Fig. 2(c). 

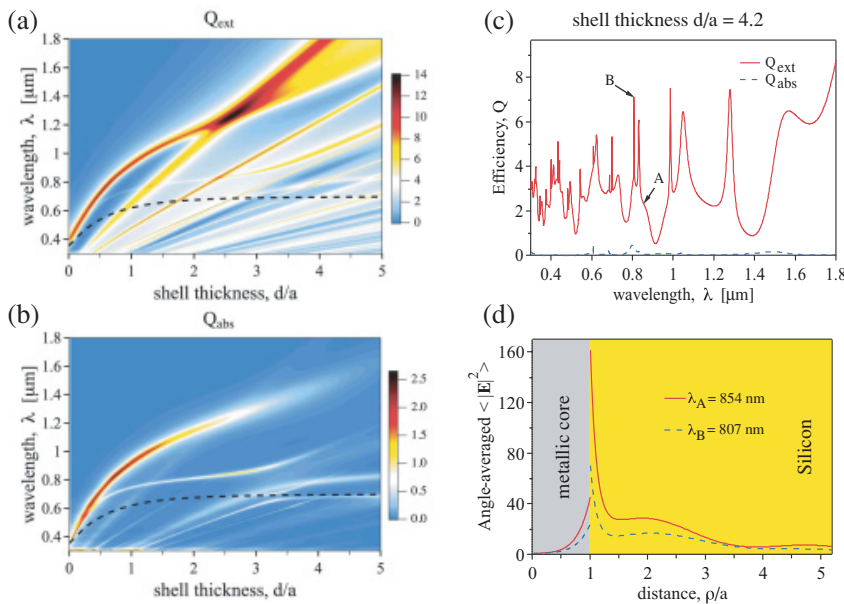

(d)

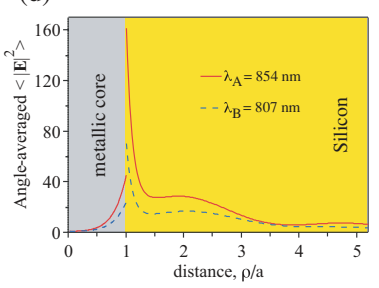

FIG. 4. (Color online) Scattering by a silver nanoparticle of radius $a=50 \mathrm{~nm}$ coated by silicon in air. (a) Extinction $Q_{\text {ext }}$ and (b) absorption $Q_{\text {abs }}$ efficiencies versus incident wavelength $\lambda$ and normalized shell thickness $d / a$. Dashed lines correspond to first-order LSPR modes for the small particle limit (2); (c) scattering for $d=$ $210 \mathrm{~nm}$ silicon coating; (d) angle-averaged electric field profile inside the nanoshell for two particular wavelengths (i) out of resonance $\lambda_{A}$ and (ii) at the resonance $\lambda_{B}$, marked in plot (c).

which can be drawn from these facts is that the resonant scattering and resonant field enhancement are not always related, and in general, should be distinguished one from another. Thus, for any particular application both properties could or should be optimized separately.

\section{B. Metallic nanoparticle coated with high-refractive-index dielectric layer}

This behavior is quite generic, and it is also applicable to the second and third geometries of silver nanoparticles coated with a dielectric layer. The results for the high-refractive-index coated dielectric layer are summarized in Fig. 4. We consider an $a=50 \mathrm{~nm}$ silver core with varying thickness of the silicon mantle in air. The extinction and absorption efficiencies in this case exhibit two types of resonances [see Figs. 4(a) and 4(b)], which can be treated in the frame of the plasmon hybridization theory [2]. One of them is associated with the LSPR's of the silver core and the second one with Mie resonances of a silicon spherical particle. They can be clearly distinguished by their dependence on the shell thickness $d$. The dielectric modes correspond to linearly redshifted Mie resonances with the thickness of the coated layer. Other types of resonances are LSPR's of the silver core, which lie below the plasma frequency, in contrast to the previous geometry [see Figs. 2(a) and 2(b)]. There is a nonmonotonous redshift of LSPR's with the shell thickness predicted by Eq. (2) [dashed lines in Figs. 4(a) and 4(b)]. Note here that the exact solution exhibits much larger shift of resonances due to the finite particle width, providing wider possibilities of the LSPR's tunability. Two types of resonances overlap at a $d=130 \mathrm{~nm}$ thickness of the coating layer leading to enhanced scattering by nanoshell particles [see Figs. 4(a) and 4(b)], although there is no enhancement of the light field inside the particle. Similar to the previous geometry, we found that maximal field
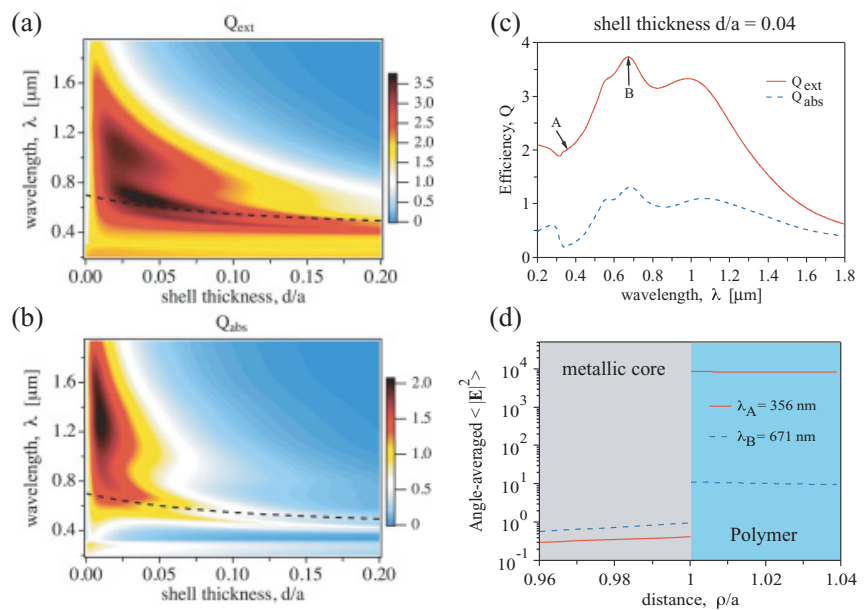

(d)

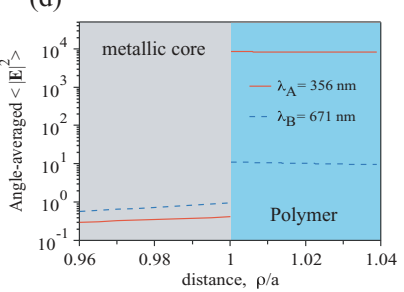

FIG. 5. (Color online) Scattering by a silver nanoparticle of radius $a=50 \mathrm{~nm}$ coated by a thin polymer layer in silicon. (a) Extinction $Q_{\text {ext }}$ and (b) absorption $Q_{\text {abs }}$ efficiencies versus incident wavelength $\lambda$ and normalized shell thickness $d / a$. Dashed lines correspond to firstorder LSPR modes for small particle limit (2); (c) scattering for $d=$ $2 \mathrm{~nm}$ polymer coating; (d) angle-averaged electric field profile inside the nanoshell for two particular wavelengths (i) out of resonance $\lambda_{A}$ and (ii) at the resonance $\lambda_{B}$, marked in plot (c).

enhancement takes place at an out-of-resonance region, which is at least two times larger than at the closely located narrow resonance peak [see Figs. 4(c) and 4(d)]. One may notice that the field is more strongly enhanced inside the silicon coating layer than inside the silver core.

\section{Metallic nanoparticle coated with low-refractive-index dielectric layer}

The last geometry which we investigate is a silver core nanoparticle coated with a thin low-refractive-index dielectric embedded into silicon. The main motivation to study this structure is to investigate the possibility of squeezing light into the lower-refractive-index dielectric with low losses. To be more realistic and closer to the possible experimental realization, we show in Fig. 5 results for an $a=50 \mathrm{~nm}$ silver core coated with a polymer $n_{P}=1.5$ embedded into the silicon. We consider a thin coated layer up to $d=10 \mathrm{~nm}$. In this case, the LSPR's lie below the plasma frequency, and they are blueshifted with the shell thickness [see Figs. 5(a) and 5(b)], contrary to the previous case with a high-refractive-index dielectric coating [see Figs. 4(a) and 4(b)], which resembles voidlike excitations of metallic nanoshells [see Figs. 3(a) and 3(b)]. The extinction efficiency is maximal for the $d=2 \mathrm{~nm}$ thickness of the coating layer [see Fig. 5(c)]. By examining the field distribution for such thickness we found that the off-resonant field enhancement is three orders of magnitude larger than that at the resonance [see Fig. 5(d)] and four orders of magnitude larger than the incident light intensity. This example clearly demonstrates the ability to squeeze the light into a subwavelength volume with a large enhancement factor. It is worth noting that because the light is concentrated mostly in the low-index dielectric layer, the losses are minimal at that point [see Fig. 5(b)]. By using a nonlinear polymer for the coating layer such local field enhancement may lead to 
a nonlinear response of the whole structure with a variety of application in photonincs, including bistability and all-optical switching.

\section{CONCLUSION}

We studied light scattering by metal-dielectric spherical particles for three geometries. One of them is a metallic nanoshell with a dielectric core and the other two are metallic nanoparticles coated by high and low-refractive-index dielectric layers. In addition to the external LSPR's, the first geometry supports internal or voidlike resonances, where the electric field is more localized inside the dielectric core. We demonstrated that scattering resonances, in general, do not correspond to resonant field enhancement of the nanoparticle. We suggested that such behavior can be interpreted in terms of the Fano resonances, which are very common in the scattering by particles. It also implies that strong field enhancement for a particular application should be sought independently from scattering resonances, although they are still linked to each other. In the case of dielectric-coated metallic nanoparticles, a field enhancement more than four orders of magnitude was achieved for the coating with a low-refractive-index dielectric layer of subwavelength thickness. The use of a nonlinear dielectric for coating in such a geometry may open up new opportunities for achieving tunable sensors or all-optical functionality for photonics applications.

\section{ACKNOWLEDGMENTS}

The author thanks Prof. Yuri Kivshar for useful discussions. The work has been supported by the Australian Research Council through the Discovery and Centre of Excellence projects.
[1] S. A. Maier, Plasmonics: Fundamentals and Applications (Springer, New York, 2007).

[2] E. Prodan, C. Radloff, N. J. Halas, and P. Nordlander, Science 302, 419 (2003).

[3] E. Prodan and P. Nordlander, J. Chem. Phys. 120, 5444 (2004).

[4] M. A. Noginov, G. Zhu, A. M. Belgrave, R. Bakker, V. M. Shalaev, E. E. Narimanov, S. Stout, E. Herz, T. Suteewong, and U. Wiesner, Nature (London) 460, 1110 (2009).

[5] D. J. Bergman and M. I. Stockman, Phys. Rev. Lett. 90, 027402 (2003).

[6] L. Lorenz, Det Kongelige Danske Videnskabernes Selskabs Skrifter 6, 1 (1890).

[7] G. Mie, Ann. Phys. (Leipzig) 330, 377 (1908).

[8] A. E. Miroshnichenko, S. Flach, A. V. Gorbach, B. S. Luk'yanchuk, Y. S. Kivshar, and M. I. Tribelsky, Opt. Phon. News 19, 48 (2008).

[9] C. Bohren and D. Huffman, Absorption and Scattering of Light by Small Particles (Wiley Interscience, New York, 1983).

[10] M. I. Tribelsky and B. S. Luk'yanchuk, Phys. Rev. Lett. 97, 263902 (2006).

[11] M. I. Tribel'skii, Soviet Physics - JETP 59, 534 (1984).

[12] A. L. Aden and M. Kerker, J. Appl. Phys. 22, 1242 (1951).
[13] see for example a list of available codes with descriptions [http://diogenes.iwt.uni-bremen.de/vt/laser/wriedt/ Mie_Type_Codes/body_mie_type_codes.html].

[14] P. B. Johnson and R. W. Christy, Phys. Rev. B 6, 4370 (1972).

[15] U. Kreibig and C. v. Fragstein, Z. Phys. A 224, 307 (1969).

[16] U. Kreibig, J. Phys. F 4, 999 (1974).

[17] T. V. Teperik, V. V. Popov, and F. J. Garcia de Abajo, Phys. Rev. B 69, 155402 (2004).

[18] D. W. Brandl and P. Nordlander, J. Chem. Phys. 126, 144708 (2007).

[19] K. Höflich, U. Gösele, and S. Christiansen, Phys. Rev. Lett. 103, 087404 (2009).

[20] J. J. Penninkhof, L. A. Sweatlock, A. Moroz, H. A. Atwater, A. van Blaaderen, and A. Polman, J. Appl. Phys. 103, 123105 (2008).

[21] C.-W. Qiu and L. Gao, J. Opt. Soc. Am. B 25, 1728 (2008).

[22] T. Kaiser, S. Lange, and G. Schweiger, Appl. Opt. 33, 7789 (1994).

[23] M. I. Tribelsky, S. Flach, A. E. Miroshnichenko, A. V. Gorbach, and Y. S. Kivshar, Phys. Rev. Lett. 100, 043903 (2008).

[24] U. Fano, Phys. Rev. 124, 1866 (1961).

[25] A. E. Miroshnichenko, S. Flach, and Y. S. Kivshar, e-print arXiv:0902.3014v4. 